\title{
The organization of project evaluation under competition
}

\author{
Peter-J. Jost · Frauke Lammers
}

Received: 8 July 2008/Accepted: 27 April 2009/Published online: 27 May 2009

(C) Springer-Verlag 2009

\begin{abstract}
This article examines the optimal organizational form of project evaluation under competition. The evaluations are carried out by two fallible screening units that sequentially assess projects. Screening can be organized as a hierarchy or a polyarchy. We show that as competitive pressure rises, the polyarchy becomes less attractive. Therefore, different organizational forms might be found in different industries depending on the degree of competition. In addition, we examine endogenous screening rules under competition: For symmetric situations, we show that polyarchies will employ higher decision thresholds compared to hierarchies. Nonetheless, as in the case of exogenous screening rules, the hierarchy becomes more attractive the higher the degree of competition.
\end{abstract}

Keywords Organization · Project evaluation · Competition

JEL classification D2 $\cdot$ L2

\section{Introduction}

Firms often depend on the implementation of new ideas in order to be successful. The evaluation of potential new projects therefore is an important activity for a firm. The seminal paper by Sah and Stiglitz (1986) considers this problem of project evaluation given that individuals are fallible in their project assessment. Thus, it may be beneficial to ask different individuals to evaluate a given project. The authors then examine two distinct organizational forms: hierarchies and polyarchies.

P.-J. Jost $(\bowtie) \cdot$ F. Lammers

WHU-Otto Beisheim School of Management, Burgplatz 2, 56179 Vallendar, Germany

e-mail: pjjost@whu.edu

F. Lammers

e-mail: frauke.lammers@whu.edu 
These forms represent different rules to aggregate the information gathered during the screening process: In a hierarchy, unanimous approval by the screening units is required for project approval. In contrast, in a polyarchy the approval of only one screening unit suffices for acceptance.

We extend this framework by considering the effect of competition on the optimal organization of project evaluation. In particular, we examine a duopolistic situation where a firm's payoff for successfully implementing a new project depends on the implementation decision of its rival. For specificity, one may think of projects arriving one by one but simultaneously at both firms. Depending on the results of their screening processes, both firms independently decide whether to implement the project or not. Three scenarios are possible: (i) both firms accept the same project for implementation resulting in a competitive outcome; (ii) only one of the two firms implements the project and derives monopoly rents; (iii) both firms reject the project.

We find that competition renders the polyarchy less attractive. Competition reduces the value a firm can expect from implementing a good project since in some cases the other firm implements this project as well. As a consequence, the advantage of a polyarchy in accepting good projects becomes less important under competition whereas the advantage of a hierarchical structure in rejecting bad projects remains unchanged. This effect is more pronounced the higher the degree of competition. In addition, we show why different organizational structures might prevail in different industries. We find that all different organizational settings might be found. The optimal organizational choice depends on the competitive pressure in the industry.

We then extend our setting by allowing endogenous screening rules. In this case the firm has to determine a decision threshold $R$ for the evaluators: A project is only accepted if its observed profit is above this reservation level. We find that the screening in a polyarchy is more conservative than that in a hierarchy (the optimal decision thresholds will be higher in a polyarchy). This result translates from the monopolistic case and is thus not changed by the introduction of competition. The intuition is that in a hierarchy both screening units know that their decisions will be rechecked at the other unit. In contrast, in a polyarchy no such rechecking occurs and some of the projects have already been rejected by the other unit. Thus the polyarchy employs higher screening standards. Nonetheless, as in the case of exogenous screening rules the hierarchy becomes more attractive the higher the degree of competition.

The Sah and Stiglitz approach has been extended in different directions. On the cost side, Koh (1992) introduces variable screening costs whereas Gersbach and Wehrspohn (1998) consider the effect of a budget constraint for project development. Swank and Visser (2008) and Jost and Lammers (forthcoming) consider the effect of agency problems when agents have reputational concerns or when one of the agents has to implement the approved projects. Gehrig et al. (2000) examine endogenous decision thresholds for each screening unit. They find that in some cases asymmetric decision rules across screening units are optimal. In contrast, in our setting with competition we focus on symmetric decision rules inside any one firm. Bull and Ordover (1987) consider a hierarchical decision-making structure and examine the effect of competition on the number of screening units. Similarly to our result, they find that screening gets tighter as the degree of competition increases. In 
their case the number of screening units increases. In contrast, in our setting the optimal organizational form of screening changes and the polyarchy becomes less attractive compared to the hierarchy.

Most closely related to our setting is Gehrig (2004). He examines a setting of R\&D with two firms acting in two distinct markets. He discusses how different forms of cooperation effect a firm's willingness to invest in information acquisition. In contrast, we consider a strictly competitive setting without cooperation.

The paper is organized as follows. In Sect. 2, we present the basic model. In Sect. 3, we investigate the effect of competition on the optimal organizational form. In Sect. 4, we examine endogenous decision thresholds under competition. Sect. 5 concludes.

\section{The basic model}

Let us consider first a market environment in which a single firm operates. This setting has been extensively analyzed starting with the seminal paper by Sah and Stiglitz (1986). The firm faces a sequence of projects with unknown value. To evaluate projects the firm can use one of the following two types of organizational forms: (1) A hierarchy with two evaluation units in which each unit screens a project and a project is approved only if both units suggest its implementation. (2) A polyarchy with two evaluation units in which a project is accepted for implementation if at least one unit recommends it.

We consider a portfolio of projects which consists only of two types of projects, good and bad projects. A good project's return is $z_{M}>0$, a bad project's return is $-z_{B}<0$. The ex ante probability that an evaluation unit faces a good project is $\alpha$. Let

$$
\beta:=\frac{\alpha z_{M}}{(1-\alpha) z_{B}}
$$

denote the quality of the project pool. $\beta=1$ then represents the case in which the portfolio is moderate, that is, the expected value of a project selected is zero. This happens, for example, if the number of good and bad projects is equal $\left(\alpha=\frac{1}{2}\right)$ and projects' returns are symmetric $\left(z_{M}=z_{B}\right)$. Hence, portfolios with $\beta<1$ have low quality with $\alpha<\frac{1}{2}$ and/or $z_{M}<z_{B}$ and those with $\beta>1$ have high quality with $\alpha>\frac{1}{2}$ and/or $z_{M}>z_{B}$.

The organizational units perform project screenings to identify the potential value of a project. However, these evaluations are done with judgement errors. On the one hand, if the project is actually good, we denote with $p_{1} \in[0,1]$ the probability that the information signals a positive return of the project, whereas with probability $\left(1-p_{1}\right)$ the screening indicates a negative return. In the last case, the screen would not recommend implementation although the project is good (Type-Ierror). On the other hand, if the real nature of the project is bad, the information signals a positive return of the project with probability $p_{2} \in[0,1]$ and with probability $\left(1-p_{2}\right)$ the information indicates a negative return. In the first case, the unit would propose the implementation of a bad project (Type-II-error). We assume that evaluation has some discriminating capability, that is $p_{1}>p_{2}$. We further 
assume that each unit observes an independent signal about the project's type and that all units are identical with respect to their screening abilities.

The conditional probability that a project is accepted for implementation then depends on the organizational form of the evaluation process. In a polyarchy the recommendation of only one unit is necessary for implementation, resulting in an acceptance rate of $p^{2}+2 p(1-p)=p(2-p)$. In a hierarchy, a project is implemented only if both units approve it, thus the acceptance rate is $p^{2}$ and lower than the one in a polyarchy.

Of course, whether it is beneficial for the organization to accept more or fewer projects depends on the quality $\beta$ of the initial portfolio. A hierarchy results in higher expected profits if the initial portfolio is sufficiently bad, whereas a polyarchy is better if the portfolio is sufficiently good. Sah and Stiglitz (1986) provide the reasoning for this finding: For a given screening function, the relative advantage of a polyarchy is in accepting good projects, whereas the relative advantage of a hierarchy is in rejecting bad projects. To see this, let

$$
\begin{aligned}
\Pi_{H} & =\alpha p_{1}^{2} z_{M}-(1-\alpha) p_{2}^{2} z_{B} \\
& =(1-\alpha) z_{B}\left(\beta p_{1}^{2}-p_{2}^{2}\right) ; \\
\Pi_{P} & =\alpha p_{1}\left(2-p_{1}\right) z_{M}-(1-\alpha) p_{2}\left(2-p_{2}\right) z_{B} \\
& =(1-\alpha) z_{B}\left(\beta p_{1}\left(2-p_{1}\right)-p_{2}\left(2-p_{2}\right)\right)
\end{aligned}
$$

be the expected profits in case of a hierarchy and a polyarchy, respectively. Then the relative advantage of a polyarchy over a hierarchy is

$$
\Pi_{P}-\Pi_{H}=2(1-\alpha) z_{B}\left(\beta p_{1}\left(1-p_{1}\right)-p_{2}\left(1-p_{2}\right)\right)
$$

and $\Pi_{H}$ is smaller than $\Pi_{P}$ if $\beta$ is sufficiently large $\left(\beta>\frac{p_{2}\left(1-p_{2}\right)}{p_{1}\left(1-p_{1}\right)}\right)$, and vice versa. ${ }^{1}$ Thus, the optimal organizational form depends on the quality of the project pool $\beta$ and on the screening technology $\frac{p_{2}\left(1-p_{2}\right)}{p_{1}\left(1-p_{1}\right)}$.

\section{Project evaluation under competition}

\subsection{Symmetric organizational forms}

We now extend the basic framework of Sect. 2 and consider a market environment with two competing firms, $A$ and $B$. Both firms face the same pool of projects and screen the projects independently. For specificity, one may think of projects arriving one by one but simultaneously at both firms. Depending on the results of their screening processes both firms independently decide whether to implement the

\footnotetext{
${ }^{1}$ In our setting the firm would never choose to have only one evaluation unit. The reason is that we do not consider evaluation costs or time to market considerations. Therefore, it always pays off to allow the possibility of a second screening: If the initial portfolio is good, the polyarchy offers the advantage to screen a project a second time that has already been declined by the first screening unit. The probability of a Type-I-error is therefore reduced. If the initial portfolio is bad, the hierarchy requires both screening units to recommend a project before it gets implemented. The probability of a costly Type-II-error thus can be reduced.
} 
project or not. Three scenarios are possible: (1) Both firms accept the same project for implementation resulting in a competitive outcome. (2) Only one of the two firms implements the project and derives monopoly rents. (3) Both firms reject the project.

How does competition affect the organizational design of project screening? To answer this question, we need to introduce competition in our setting. The effect of competition may depend on several factors such as the number of competitors, whether competition is in prices or quantities and how much products are differentiated. We take a very general setup as in Schmidt (1997) where the degree of competition is expressed through its effect on the profit function of the firm: If competition increases, profits go down. In particular, let $z_{M}$ as before be the monopoly rent if only one firm implements a good project and let $z_{D}$ be the value of the good project under competition, that is, if both firms implement the same project. We assume that competition reduces firms' revenue from projects compared to a monopoly situation, that is, $0 \leq z_{D} \leq z_{M}$. If both firms act in the same market and products are perfect substitutes, we have $z_{D} \leq z_{M} / 2$ that is, the best situation for both firms in the duopolistic case is when monopoly rents are equally split. If both firms act in independent markets or products are not perfect substitutes, we could have $z_{D} \geq z_{M} / 2$. In the extreme, if $z_{D}=z_{M}$, a successful implementation of a project by one firm in one market does not influence the value of this project for the second firm in its home market.

Let

$$
\gamma:=\frac{\alpha\left(z_{M}-z_{D}\right)}{(1-\alpha) z_{B}}
$$

denote the reduced quality of the project pool under competition if a firm receives the duopoly value of a project instead of its monopoly rents. Through the parameter $z_{D}, \gamma$ can also be seen as a measure of the degree of competition. If competition reduces the value of good projects to zero, i.e. $z_{D}=0$, then $\gamma=\beta$ and all monopoly rents are lost if both firms implement the project. If the monopoly rent is split under competition, $z_{D}=z_{M} / 2$, the quality of the project pool under competition is also split and we have $\gamma=\beta / 2$. And finally, if firms act in completely independent markets, $z_{D}=z_{M}$, competition does not influence the expected value of projects, $\gamma=0$.

This setting can also be related to a duopolistic setup with differentiated goods, as for example in Schmutzler (2007). Here the degree of competition $\left(z_{D}\right.$ and the resulting $\gamma$ ) corresponds to a measure of substitutability of the goods. In a richer oligopolistic setting one could also think of $z_{D}$ (and $\gamma$ ) as a measure of the number of firms active in the market. That is, the more firms compete, the lower will be $z_{D}$ and the higher $\gamma$.

First, we consider symmetric organizational forms where both firms choose the same organizational structure. That is, either both firms are organized as a hierarchy or both are organized as a polyarchy.

Proposition 1 For symmetric organizational forms, competition renders the polyarchy less attractive. 
Proof Denote as $\Pi_{i j}, i, j \in\{H, P\}$ the expected profit of firm $i$ choosing organizational form $i$ given that its competitor is organized according to $j$. For symmetric organizational forms we have:

$$
\begin{aligned}
\Pi_{H H} & =\alpha p_{1}^{2}\left(p_{1}^{2} z_{D}+\left(1-p_{1}^{2}\right) z_{M}\right)-(1-\alpha) p_{2}^{2} z_{B} \\
\Pi_{P P} & =\alpha p_{1}\left(2-p_{1}\right)\left(p_{1}\left(2-p_{1}\right) z_{D}+\left(1-p_{1}\left(2-p_{1}\right)\right) z_{M}\right) \\
& -(1-\alpha) p_{2}\left(2-p_{2}\right) z_{B} .
\end{aligned}
$$

Under a hierarchical structure, for example, the ex ante probability that both firms implement a good project is given by $p_{1}^{2} p_{1}^{2}$ while the probability that only one firm accepts this project for implementation is $p_{1}^{2}\left(1-p_{1}^{2}\right)$. Simple calculation then shows that the relative advantage of a polyarchical structure over a hierarchy in case of competition is

$$
\Pi_{P P}-\Pi_{H H}=2(1-\alpha) z_{B}\left(\beta p_{1}\left(1-p_{1}\right)-p_{2}\left(1-p_{2}\right)-2 \gamma p_{1}^{2}\left(1-p_{1}\right)\right),
$$

hence the difference to the relative advantage in case of monopoly, see Eq. (1), is

$$
\left(\Pi_{P P}-\Pi_{H H}\right)-\left(\Pi_{P}-\Pi_{H}\right)=-4 \alpha p_{1}^{2}\left(1-p_{1}\right)\left(z_{M}-z_{D}\right)
$$

which is always negative.

The intuition for this result is that competition reduces the value a firm can expect from implementing a good project. This is because in some cases the other firm implements this project as well. As a consequence, the advantage of a polyarchy in accepting good projects becomes less important under competition whereas the advantage of a hierarchical structure in rejecting bad projects remains unchanged. Hence, a hierarchy performs better than a polyarchy under competition than under monopoly and this advantage is higher, the higher the degree of competition.

The influence of the market environment on the relative advantage of polyarchies over hierarchies is summarized in Fig. 1 for the case that the quality of the project pool is moderate, $\beta=1$. If both firms act in completely independent markets, $z_{D}=z_{M}(\gamma=0)$, competition has no effect on the optimal choice of the organizational form, see Eq. (3). Using Eq. (1), we immediately see that a polyarchy results in higher expected profits if $p_{2}<1-p_{1}$, that is, in the area $0 B_{0} X_{1}$. Increasing competition, that is lowering $z_{D}$, implies increasing $\gamma$ and the symmetric polyarchical structure becomes less beneficial compared to hierarchical structures, see Eq. (2), and is better only in the area $0 B_{\gamma} X_{1}$ for $\gamma<\frac{1}{2}$, respectively $0 B_{\gamma} X_{\frac{1}{2 \gamma}}$ for $\gamma>\frac{1}{2}$. In the extreme case in which competition reduces the competitive outcome to $z_{D}=0$ and $\gamma=1$, a symmetric polyarchy is better than a symmetric hierarchy only in the area $0 B_{1} X_{\frac{1}{2}}$.

The picture changes slightly, if the project pool is better or worse, that is, $\beta \neq 1$ (Fig. 2). Using Eq. (3), we know that for $z_{D}=z_{M}$ competition has no effect on relative advantages and, by Eq. (1), a lower quality of the project pool successively diminishes the area for which a symmetric polyarchy performs better than hierarchical structures to $0 B_{0}^{\prime} X_{1}$. As before, this area shrinks as competition increases. If competition revenues are zero, $z_{D}=0$ and $\gamma=\beta$, the area is $0 B_{\beta}^{\prime} X_{\beta}$ 


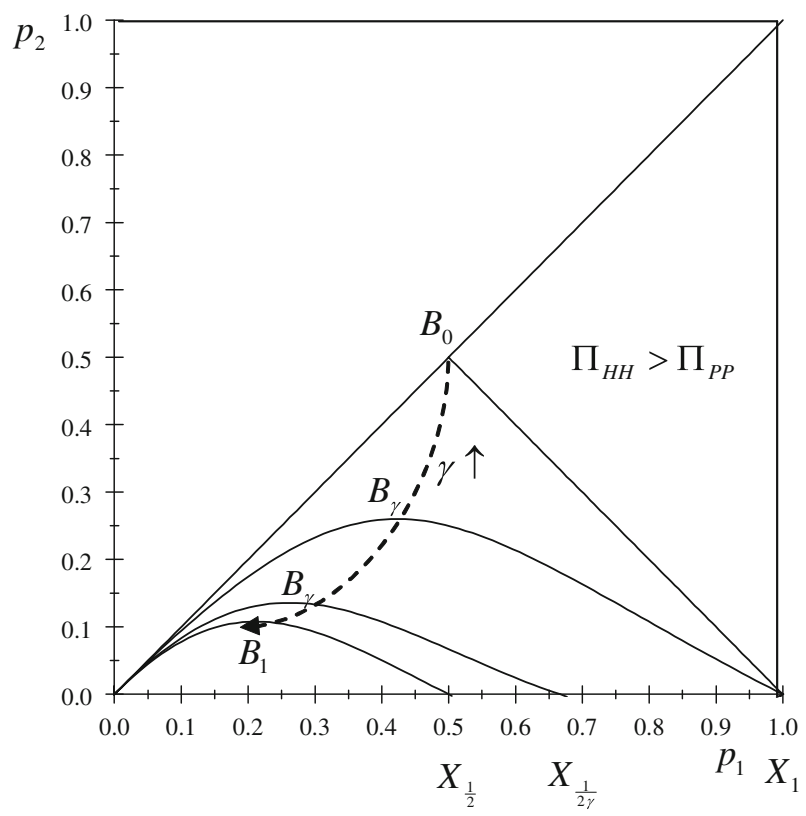

Fig. 1 Advantage of polyarchy over hierarchy for varying degrees of competition (with $\beta=1$ )

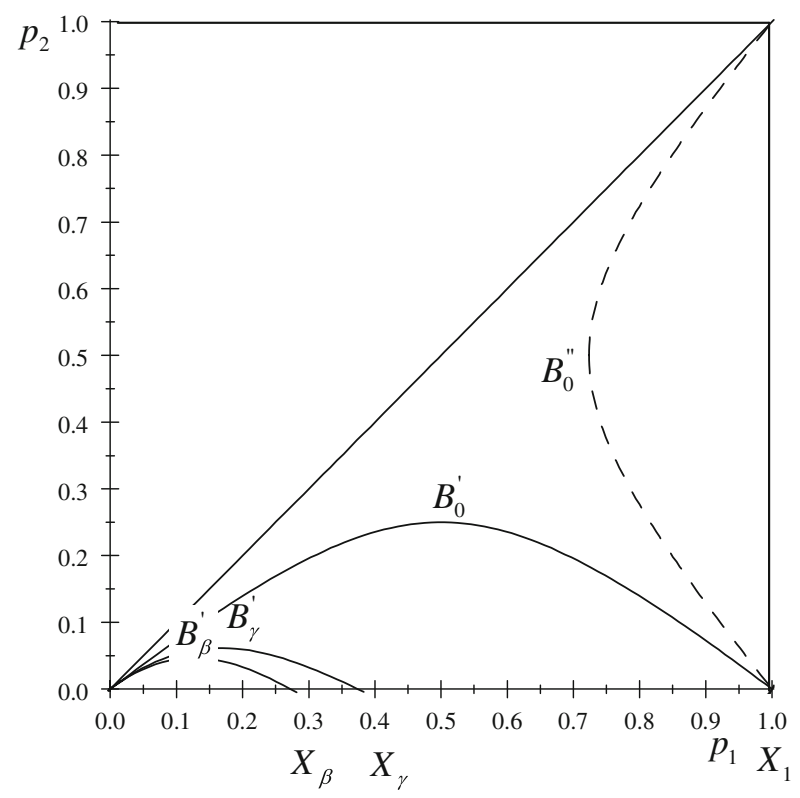

Fig. 2 Advantage of polyarchy over hierarchy for different degrees of project quality $(\beta \neq 1)$ 
where polyarchy dominates hierarchy is smallest. The reverse holds if the project pool is good, that is, $\beta>1$. In this case, polyarchical structures are better than hierarchical ones outside the area $X_{1} B_{0}^{\prime \prime} 1$.

Our findings are of a similar nature to those in Bull and Ordover (1987). These authors consider a purely hierarchical decision-making structure and examine the effect of competition on the number of screening units. They show that as the expected return of projects falls, e.g. due to competition or different cost structures, the number of screening units increases and thus screening gets tighter. In contrast, in our setting screening gets tighter through a change in the optimal organizational form, i.e., the polyarchy becomes less attractive compared to the hierarchy.

Decision making procedures inside firms are often difficult to assess from the outside. However, our result that the polyarchy becomes less attractive as competitive pressure rises, seems to be consistent with evidence from practice. For instance, in Germany decision-making responsibilities of the Supervisory Board have changed considerably over the last 10 years. As competitive pressure has risen due to the globalization and technological change, the role of the Supervisory Board has changed. Once the focus was on pure monitoring, whereas nowadays the role has changed to co-decision-making, where Management and Supervisory Board both have to agree to important decisions (Cromme 2005).

\subsection{Equilibrium organizational forms}

Of course, firms can decide to organize their screening differently. To analyze the optimal organizational form we consider the following course of interactions: In a first stage firms simultaneously choose the organizational form of their screening process. Within this organizational form firms simultaneously screen a sequence of projects in the second stage. Depending on their evaluations firms implement those projects accepted by their screening units.

The following proposition summarizes the equilibrium organizational structures:

Proposition 2 In case of competition the following organizational forms are optimal. If

1. $\gamma>\frac{1}{p_{1}^{2}}\left(\beta-\frac{p_{2}\left(1-p_{2}\right)}{p_{1}\left(1-p_{1}\right)}\right),(H, H)$ is the optimal organizational form, and

$$
\Pi_{H H}>\Pi_{P P}
$$

2. $\quad \gamma \in\left(\frac{1}{p_{1}\left(2-p_{1}\right)}\left(\beta-\frac{p_{2}\left(1-p_{2}\right)}{p_{1}\left(1-p_{1}\right)}\right), \frac{1}{p_{1}^{2}}\left(\beta-\frac{p_{2}\left(1-p_{2}\right)}{p_{1}\left(1-p_{1}\right)}\right)\right)$, there exist two asymmetric equilibria $(P, H)$ and $(H, P)$, and

$$
\Pi_{P H}>\Pi_{H P}
$$

3. $\gamma<\frac{1}{p_{1}\left(2-p_{1}\right)}\left(\beta-\frac{p_{2}\left(1-p_{2}\right)}{p_{1}\left(1-p_{1}\right)}\right),(P, P)$ is the optimal organizational form, and

$$
\begin{array}{ll}
\Pi_{P P}>\Pi_{H H} & \text { if } \gamma<\frac{1}{2 p_{1}}\left(\beta-\frac{p_{2}\left(1-p_{2}\right)}{p_{1}\left(1-p_{1}\right)}\right) ; \\
\Pi_{P P}<\Pi_{H H} & \text { otherwise. }
\end{array}
$$


Proof see Appendix The Proposition shows why different organizational structures might prevail in different industries. The optimal organizational choice now depends not only on the screening technology $\frac{p_{2}\left(1-p_{2}\right)}{p_{1}\left(1-p_{1}\right)}$ and the quality of the initial project pool $\beta$, but also on the competitive pressure in the industry. If the firms operate in distinct markets $\left(z_{D}=z_{M} \Rightarrow \gamma=0\right)$, we are back in the monopoly case of Sect. 2 with $\beta>$ reqqless $\frac{p_{2}\left(1-p_{2}\right)}{p_{1}\left(1-p_{1}\right)}$ determining the optimal organization. For $\beta<\frac{p_{2}\left(2-p_{2}\right)}{p_{1}\left(2-p_{1}\right)}$ the hierarchy is the optimal organizational form in the monopolistic case, and since we have $\gamma \geq 0$, the hierarchy stays optimal under competition.

In contrast, consider in the following the case where $\frac{p_{2}\left(1-p_{2}\right)}{p_{1}\left(1-p_{1}\right)}<\beta$. Then, without competition the firm would organize as a polyarchy. However, with competition this does not need to be optimal any longer. As competitive pressure rises, the optimal organizational form changes: The polyarchy becomes less attractive since monopoly rents are no longer earned for sure. Therefore, the stronger the competition (low values $z_{D} \Rightarrow$ high $\gamma$ ), the more likely is that both firms will organize as a hierarchy.

For a market with intermediate competitive pressure, we have the case that one firm chooses the hierarchy and the other firm the polyarchy as organizational form. Then, the polyarchy earns higher expected profits. The reason is that in this case a monopolistic firm would choose to organize as a polyarchy. Accepting projects is therefore per se attractive. The polyarchy will accept more projects than the hierarchy since the decision rule is less restrictive. Therefore, the polyarchy is more often in the position to be the only firm in the market to accept a given project and is therefore more often able to earn monopoly rents.

In this case we could also see a monopolist preventing entry from a rival firm if this entry would entail an intermediate competitive pressure. The monopolist, being the first to choose his organizational form and deciding to organize as a polyarchy, would earn higher expected profits then the rival firm. For $\Pi_{P H}>0$ and $\Pi_{H P}<0$, the potential rival would thus refrain from entering the industry. As a consequence the monopolist would earn even higher expected profits $\Pi_{P}>\Pi_{P H}$.

For industries with little competition, both firms should choose a polyarchical structure. However, as the competitive pressure rises slightly, we have $\Pi_{P P}<\Pi_{H H}$ and the firms would be better off if they could commit to both organizing as a hierarchy. However, this commitment is hard to enforce. Each firm would have a strong incentive to deviate to a polyarchy in order to earn monopoly rents more often. Since the organizational structure is hard to monitor from the outside, cooperation would be threatened to fail. In situations with a low to medium competitive pressure we thus have the classic situation of a Prisoners' Dilemma: Both firms could gain from cooperation, however, both have an incentive to defect.

\section{Endogenous screening rules}

The screening units in the above model receive a binary imperfect signal with regard to the quality of the projects. In this setting $p_{1}\left(p_{2}\right)$ is the exogenous probability that the information signals a positive return given the project is good (bad). In a more general case, the units will receive a richer set of signals that has to 
be processed. In this case each firm has to decide under what conditions the screening unit should approve a given project for implementation.

For instance, a unit observes a signal $y=z+\theta$ of the project's monopolistic return $z$ and an independently distributed error $\theta$ with distribution function $M(\theta)$. As before we consider two types of projects: good and bad. We follow Sah and Stiglitz (1986) and assume that the screening units can only communicate binary information, that is, whether they think that a project is good or bad. Therefore the firm has to determine a decision threshold $R$ for the evaluators: A project is only accepted if its observed profit is above this reservation level. ${ }^{2}$ The endogenous screening function $p(z, R)$ is then given by

$$
p(z, R):=\operatorname{prob}(y \geq R)=1-M(R-z) .
$$

We have $\frac{\partial p(z, R)}{\partial R}=p^{\prime}(R) \leq 0$. The higher the decision threshold, the lower the probability that a given project is accepted. We denote the probability to accept a good project with $p_{1}(R)=p\left(z=z_{M}, R\right)$ and the probability to accept a bad project $p_{2}(R)=p\left(z=z_{B}, R\right)$.

\subsection{Optimal decision thresholds under competition}

We consider the same competitive environment as described in Sect. 3. In a first step both firms simultaneously decide on their organizational form. They are then able to observe each other's decision. What is new compared to Sect. 3 is a second step where both firms simultaneously choose their respective decision threshold. ${ }^{3}$

As in Sah and Stiglitz (1986) we assume that the profit functions $\Pi_{i j}$ with $i, j \in\{H, P\}$ are concave with respect to $R .^{4}$ Then, the optimal decision threshold $R^{i j}$ for firm $i$ given the organizational form and the decision threshold of firm $j$ has to solve:

$$
\begin{aligned}
& \frac{\partial \Pi_{H H}}{\partial R^{H H}}=0 \Leftrightarrow\left(\beta-\gamma p_{1}^{2}\right) p_{1}^{\prime}\left(R^{H H}\right) p_{1}\left(R^{H H}\right)=p_{2}^{\prime}\left(R^{H H}\right) p_{2}\left(R^{H H}\right) ; \\
& \frac{\partial \Pi_{P P}}{\partial R^{P P}}=0 \Leftrightarrow\left(\beta-\gamma p_{1}\left(2-p_{1}\right)\right) p_{1}^{\prime}\left(R^{P P}\right)\left(1-p_{1}\left(R^{P P}\right)\right)=p_{2}^{\prime}\left(R^{P P}\right)\left(1-p_{2}\left(R^{P P}\right)\right) ; \\
& \frac{\partial \Pi_{H P}}{\partial R^{H P}}=0 \Leftrightarrow\left(\beta-\gamma p_{1}\left(2-p_{1}\right)\right) p_{1}^{\prime}\left(R^{H P}\right) p_{1}\left(R^{H P}\right)=p_{2}^{\prime}\left(R^{H P}\right) p_{2}\left(R^{H P}\right) \\
& \frac{\partial \Pi_{P H}}{\partial R^{P H}}=0 \Leftrightarrow\left(\beta-\gamma p_{1}^{2}\right) p_{1}^{\prime}\left(R^{P H}\right)\left(1-p_{1}\left(R^{P H}\right)\right)=p_{2}^{\prime}\left(R^{P H}\right)\left(1-p_{2}\left(R^{P H}\right)\right)
\end{aligned}
$$

For $\gamma=0$ both firms operate in separate markets and we are in the monopolistic situation. For $\gamma>0$ the following proposition shows comparative statics of the competitive decision thresholds.

\footnotetext{
${ }^{2}$ See also Gehrig et al. (2000) who consider endogenous screening rules for each screening unit separately.

3 The timing of the interaction is based on the assumption that the organizational form is a more longterm decision compared to the definition of the decision threshold.

${ }^{4}$ See Sah and Stiglitz, Footnote 7. The profit functions under competition $\Pi_{i j}$ are given in the Appendix.
} 
Proposition 3 In a competitive environment the following result holds irrespective of the organizational forms of both firms: An increase in the competitive pressure in the industry (decrease in $z_{D}$ ) increases the decision threshold. That is $\frac{\partial R^{i j}}{\partial z_{D}}<0$ for $i, j \in\{H, P\}$.

Proof see Appendix The intuition behind this result can be related to the monopolistic setting: A stronger competitive pressure translates to a reduced payoff from successfully implementing a good project. At the same time the loss from implementing a bad project remains unchanged. Therefore, a stronger competition has a similar effect as a decrease in the good project's return. Fewer projects should be accepted and thus the decision threshold should be higher.

Proposition 4 For symmetric organizational forms, the decision thresholds will be higher for polyarchies compared to hierarchies, that is $R^{P P}>R^{H H}$.

Proof see Appendix The screening in a polyarchy is more conservative than that in a hierarchy. This result from the monopolistic case is thus not changed by the introduction of competition. ${ }^{5}$ The intuition is that in a hierarchy the first screening unit knows that its decision to approve a project is rechecked at the second unit. And the second unit knows that all projects it receives have already received a favorable assessment. In contrast, in a polyarchy each unit knows that its decision will not be rechecked. In addition, some of the projects have already been rejected by the other unit. To reflect these differences, the optimal decision thresholds will be higher in a polyarchy.

\subsection{Effect of competition on the optimal organization}

We now examine the optimal organizational form with endogenous screening rules under competition. Define $k:=\left(\frac{p_{1}^{\prime}}{p_{1}}\right) /\left(\frac{p_{2}^{\prime}}{p_{2}}\right)$. For the monopolistic situation it can be shown that for $k \geq 1$ the hierarchy performs better than the polyarchy. ${ }^{6}$ For competition we can derive the following result:

Proposition 5 For symmetric organizational forms with endogenous screening


$\frac{z_{M}-\left(z_{M}-z_{D}\right) 2 p_{1}}{z_{M}-\left(z_{M}-z_{D}\right) p_{1}\left(2-p_{1}\right)}$.

Proof From (4) we have $\left(\beta-\gamma p_{1}\left(2-p_{1}\right)\right) p_{1}^{\prime}\left(R^{P P}\right)\left(1-p_{1}\left(R^{P P}\right)\right)=p_{2}^{\prime}\left(R^{P P}\right)$ $\left(1-p_{2}\left(R^{P P}\right)\right)$. Using the definition of $k$ at $R=R^{P P}$ we get

$$
k\left(\beta-2 \gamma p_{1}+\gamma p_{1}^{2}\right) p_{1}\left(1-p_{1}\right)-p_{2}\left(1-p_{2}\right)=0 .
$$

For symmetric organizational forms the hierarchy performs better than the polyarchy if (see Eq. (8) in the Appendix):

$$
\left(\beta-2 \gamma p_{1}\right) p_{1}\left(1-p_{1}\right)-p_{2}\left(1-p_{2}\right)<0 .
$$

\footnotetext{
5 For the monopolistic case see Sah and Stiglitz (1986), Proposition 4.

${ }^{6}$ Follwing Sah and Stiglitz (1986), Proposition 7.
} 
Therefore, for $k \geq \frac{\beta-2 \gamma p_{1}}{\beta-2 \gamma p_{1}+\gamma p_{1}^{2}}=\frac{z_{M}-\left(z_{M}-z_{D}\right) 2 p_{1}}{z_{M}-\left(z_{M}-z_{D}\right) p_{1}\left(2-p_{1}\right)}$, the hierarchy performs better than the polyarchy. Note that $\frac{{ }^{1} \beta-2 \gamma p_{1}}{\beta-2 \gamma p_{1}+\gamma p_{1}^{2}}<1$.

Under monopoly, that is for $z_{D}=z_{M}$ and $\gamma=0$, the necessary condition for the hierarchy to outperform the polyarchy is $k \geq 1$, as in Sah and Stiglitz. Under competition, Proposition 5 shows that the hierarchy becomes more attractive the higher the degree of competition (the lower $z_{D}$ ), that is

$$
\frac{\partial}{\partial z_{D}}\left(\frac{z_{M}-\left(z_{M}-z_{D}\right) 2 p_{1}}{z_{M}-\left(z_{M}-z_{D}\right) p_{1}\left(2-p_{1}\right)}\right)>0 \text {. }
$$

This result mimics our findings in Proposition 1.

\section{Conclusion}

How does competition effect the optimal organization of project evaluation? Using the framework by Sah and Stiglitz (1986) we explored this question in a simple duopoly model in which firms independently decide whether to evaluate projects in a hierarchical or polyarchical screening process. We found that competition in principle makes the hierarchy more attractive than the polyarchy although for an intermediate degree of competitive pressure organizational structures might be asymmetric.

In the present framework we have assumed that both firms have identical screening technologies such that evaluations are done with the same judgement errors. The introduction of heterogenous firm-specific screening functions would modify our findings without changing our main results. As in the case of a monopolistic firm a lower Type-I-error or a higher Type-II-error renders the polyarchy less attractive. However, although this effect of heterogenous screening functions influences the thresholds under which organizational forms are optimal, the pattern of equilibrium structures remains unchanged.

The present model does not examine time to market considerations. Time to market measures the length of time it takes from product research until the project is available for sale. Then, the firm that is first to introduce the product to the market is able to realize monopolistic rents for some time and could create a first mover advantage. This consideration is especially important in industries where products are outmoded quickly. When the follower enters the market he may only have a short time left to realize profits. If screenings are extensive and time-consuming, time to market considerations would favor the polyarchy since some projects are already implemented after the first screening.

Another extension of our model would be the introduction of oligopolistic industry structures. However, changing the degree of competitive pressure in the duopoly case is similar to changing the number of firms under otherwise identical environmental market conditions: The value of good projects decreases the more firms implement them. Hence, an increase in the number of firms in an industry makes hierachical project evaluation more attractive. 
In line with the paper by Gehrig (2004) it would be interesting to discuss how different forms of cooperations between firms would affect the optimal organizational form. In particular, if the competitive pressure was low such that firms organize polyarchical in equilibrium although they would jointly benefit from hierarchical structures, cooperation would be profitable. The question which form of cooperation induces joint profit maximization under these circumstances is scope for future research.

\section{Appendix}

Proof of Proposition 2 To prove this proposition, let $\Pi_{i j}$ be the expected profit of a firm that chooses an organizational structure $i \in\{H, P\}$ whereas its competitor decided for an organizational structure $j \in\{H, P\}$. Then

$$
\begin{aligned}
\Pi_{H H} & =\alpha p_{1}^{2}\left(p_{1}^{2} z_{D}+\left(1-p_{1}^{2}\right) z_{M}\right)-(1-\alpha) p_{2}^{2} z_{B} \\
& =(1-\alpha) z_{B}\left(\beta p_{1}^{2}-p_{2}^{2}-\gamma p_{1}^{4}\right) \\
\Pi_{H P} & =\alpha p_{1}^{2}\left(p_{1}\left(2-p_{1}\right) z_{D}+\left(1-p_{1}\left(2-p_{1}\right)\right) z_{M}\right)-(1-\alpha) p_{2}^{2} z_{B} \\
& =(1-\alpha) z_{B}\left(\beta p_{1}^{2}-p_{2}^{2}-\gamma p_{1}^{3}\left(2-p_{1}\right)\right) \\
\Pi_{P H} & =\alpha p_{1}\left(2-p_{1}\right)\left(p_{1}^{2} z_{D}+\left(1-p_{1}^{2}\right) z_{M}\right)-(1-\alpha) p_{2}\left(2-p_{2}\right) z_{B} \\
& =(1-\alpha) z_{B}\left(\beta p_{1}\left(2-p_{1}\right)-p_{2}\left(2-p_{2}\right)-\gamma p_{1}^{3}\left(2-p_{1}\right)\right) \\
\Pi_{P P} & =\alpha p_{1}\left(2-p_{1}\right)\left(p_{1}\left(2-p_{1}\right) z_{D}+\left(1-p_{1}\left(2-p_{1}\right)\right) z_{M}\right)-(1-\alpha) p_{2}\left(2-p_{2}\right) z_{B} \\
& =(1-\alpha) z_{B}\left(\beta p_{1}\left(2-p_{1}\right)-p_{2}\left(2-p_{2}\right)-\gamma p_{1}^{2}\left(2-p_{1}\right)^{2}\right)
\end{aligned}
$$

We have :

$$
\begin{gathered}
\Pi_{P P}-\Pi_{H H}=2(1-\alpha) z_{B}\left(\beta p_{1}\left(1-p_{1}\right)-p_{2}\left(1-p_{2}\right)-2 \gamma p_{1}^{2}\left(1-p_{1}\right)\right) \\
\Pi_{P P}-\Pi_{H P}=2(1-\alpha) z_{B}\left(\beta p_{1}\left(1-p_{1}\right)-p_{2}\left(1-p_{2}\right)-\gamma p_{1}^{2}\left(1-p_{1}\right)\left(2-p_{1}\right)\right) \\
\Pi_{P H}-\Pi_{H H}=2(1-\alpha) z_{B}\left(\beta p_{1}\left(1-p_{1}\right)-p_{2}\left(1-p_{2}\right)-\gamma p_{1}^{3}\left(1-p_{1}\right)\right)
\end{gathered}
$$

Hence

$$
\Pi_{P H}-\Pi_{H H}>\Pi_{P P}-\Pi_{H P}>\Pi_{P P}-\Pi_{H H} .
$$

1. Suppose that $\Pi_{H H}>\Pi_{P H}$. Then $\Pi_{H P}>\Pi_{P P}$ and $\Pi_{H H}>\Pi_{P P}$. Therefore, $H$ is a best response to $H$ and $P$. Symmetry implies that $(H, H)$ then is the unique equilibrium and Pareto-dominates $(P, P)$. We have $\Pi_{H H}>\Pi_{P H}$ if $\left(\beta-\gamma p_{1}^{2}\right) p_{1}\left(1-p_{1}\right)<p_{2}\left(1-p_{2}\right)$.

2. Suppose that $\Pi_{H H}<\Pi_{P H}$ and $\Pi_{H P}>\Pi_{P P}$. Hence $P$ is a best response to $H$ and $H$ is a best response to $P$. We have $\Pi_{H H}<\Pi_{P H}$ if $\left(\beta-\gamma p_{1}^{2}\right) p_{1}(1-$ $\left.p_{1}\right)>p_{2}\left(1-p_{2}\right)$ and $\Pi_{H P}>\Pi_{P P}$ if $\left(\beta-\gamma p_{1}\left(2-p_{1}\right)\right) p_{1}\left(1-p_{1}\right)<p_{2}(1-$ $\left.p_{2}\right)$. Therefore, we have $\Pi_{H P}<\Pi_{P H}$ since $p_{2}\left(1-p_{2}\right)<\beta p_{1}\left(1-p_{1}\right)$. 
3. Suppose that $\Pi_{H P}<\Pi_{P P}$. Then $\Pi_{H H}<\Pi_{P H}$. Therefore, $P$ is a best response to $P$ and $H . \Pi_{H P}<\Pi_{P P}$ if $\left(\beta-\gamma p_{1}\left(2-p_{1}\right)\right) p_{1}\left(1-p_{1}\right)>p_{2}\left(1-p_{2}\right)$. However, we have $\Pi_{P P}>\Pi_{H H}$ only for $\left(\beta-2 \gamma p_{1}\right) p_{1}\left(1-p_{1}\right)>p_{2}\left(1-p_{2}\right)$. If the reverse is true, coordination offers an improvement potential since $\Pi_{P P}<\Pi_{H H}$.

Proof of Proposition 3 We examine the effect of a change in the competitive pressure on the decision threshold. For example, consider the symmetric case where both firms organize as a hierarchy. From (4) we have $f\left(z_{D}, R^{H H}\right)=$ $\frac{\alpha}{(1-\alpha) z_{B}}\left(z_{M}-\left(z_{M}-z_{D}\right) p_{1}\left(R^{H H}\right)^{2}\right) p_{1}^{\prime}\left(R^{H H}\right) p_{1}\left(R^{H H}\right)-p_{2}^{\prime}\left(R^{H H}\right) p_{2}\left(R^{H H}\right)=0$ and for $R^{H H}$ to be a maximum we must have $\frac{\partial f}{\partial R^{H H}}<0$. We have

$$
\frac{\partial R^{H H}}{\partial z_{D}}=-\frac{\frac{\partial f}{\partial z_{D}}}{\frac{\partial f}{\partial R^{H H}}}=-\frac{\alpha}{(1-\alpha) z_{B}} \frac{p_{1}\left(R^{H H}\right)^{3} p_{1}^{\prime}\left(R^{H H}\right)}{\frac{\partial f}{\partial R^{H H}}}<0 .
$$

since $p^{\prime}(R)<0$. The same reasoning applies to the other competitive organizational forms in (4).

Therefore, the stronger the competitive pressure (small $z_{D}$ ), the higher will be the optimal decision threshold.

Proof of Proposition 4 To see that $R^{P P}>R^{H H}$, define $c(z):=\frac{1-p(z)}{p(z)}$ (i.e. $c\left(z_{M}\right)=$ $\frac{\left(1-p_{1}\right)}{p_{1}}$ and $c\left(z_{B}\right)=\frac{\left(1-p_{2}\right)}{p_{2}}$.) Calculate

$$
\begin{aligned}
c(0) \frac{\partial \Pi_{H H}}{\partial R}-\frac{\partial \Pi_{P P}}{\partial R}= & 2 \alpha p_{1}^{\prime}(R) p_{1}\left(c(0)-c\left(z_{M}\right)\right)\left(z_{M}-p_{1}^{2}\left(z_{M}-z_{D}\right)\right) \\
& +2 \alpha p_{1}^{\prime}(R) p_{1} 2 p_{1}\left(z_{M}-z_{D}\right) c\left(z_{M}\right)\left(1-p_{1}\right) \\
& -2(1-\alpha) p_{2} p_{2}^{\prime}(R) z_{B}\left(c(0)-c\left(z_{B}\right)\right) .
\end{aligned}
$$

We have $c^{\prime}(z)<0$ and therefore $c(0)-c\left(z_{M}\right)>0$ since $z_{M}>0$. We also have $p^{\prime}(R)<0$, therefore the first and the second term are negative. We have $c(0)-$ $c\left(z_{B}\right)<0$ since $z_{B}<0$. We also have $p^{\prime}(R)<0$, therefore the third term is also negative. Assume to the contrary that $R^{H H}>R^{P P}$. Then from $\frac{\partial \Pi_{P P}}{\partial R}>c(0) \frac{\partial \Pi_{H H}}{\partial R}$ it follows that for $R^{H H}>R^{P P}$ :

$$
\Pi_{P P}\left(R^{H H}\right)-\Pi_{P P}\left(R^{P P}\right)>c\left(0, R^{H H}\right) \Pi_{H H}\left(R^{H H}\right)-c\left(0, R^{P P}\right) \Pi_{H H}\left(R^{P P}\right) .
$$

Since $c(0, R)=\frac{M(R)}{1-M(R)}$, we have $\frac{\partial}{\partial R} c(0, R)=\frac{m(R)}{(1-M(R))^{2}}>0$ and thus $c\left(0, R^{H H}\right)$ $>c\left(0, R^{P P}\right)$. Therefore,

$$
\begin{aligned}
& c\left(0, R^{H H}\right) \Pi_{H H}\left(R^{H H}\right) \\
& \quad-c\left(0, R^{P P}\right) \Pi_{H H}\left(R^{P P}\right)>c\left(0, R^{P P}\right)\left(\Pi_{H H}\left(R^{H H}\right)-\Pi_{H H}\left(R^{P P}\right)\right) .
\end{aligned}
$$

Hence, for $R^{H H}>R^{P P}$ we must have

$$
\Pi_{P P}\left(R^{H H}\right)-\Pi_{P P}\left(R^{P P}\right)>c\left(0, R^{P P}\right)\left(\Pi_{H H}\left(R^{H H}\right)-\Pi_{H H}\left(R^{P P}\right)\right) .
$$

The LHS is negative since the optimal $R$ is $R^{P P}$. The RHS is positive since the optimal $R$ is $R^{H H}$ and $c\left(0, R^{P P}\right)>0$. This contradicts $R^{H H}>R^{P P}$. 


\section{References}

Bull C, Ordover JA (1987) Market structure and optimal management organizations. Rand J Econ $18: 480-491$

Cromme G (2005) Corporate Governance in Germany and the German Corporate Governance Code. Corp Gov 13:362-367

Gehrig TP (2004) Organizational form and information acquisition. J Inst Theor Econ 160:1-13

Gehrig TP, Regibeau P, Rockett K (2000) Project evaluation and organizational form. Rev Econ Des 5:177-199

Gersbach H, Wehrspohn U (1998) Organizational design with a budget constraint. Rev Econ Des 3:149_ 157

Jost PJ, Lammers F (forthcoming) Organization of project evaluation and implementation under moral hazard. Journal of Institutional and Theoretical Economics

Koh WTH (1992) Variable evaluation costs and the design of fallible hierarchies and polyarchies. Econ Lett 38:313-318

Sah RK, Stiglitz JE (1986) The architecture of economic systems: hierarchies and polyarchies. Am Econ Rev 76:716-727

Schmidt KM (1997) Managerial incentives and product market competition. Rev Econ Stud 64:191-213

Schmutzler A (2007) The relation between competition and innovation-why is it such a mess? Working paper

Swank O, Visser B (2008) The consequences of endogenizing information for the performance of a sequential decision procedure. J Econ Behav Org 65:667-681 